through a range of initiatives. For more information, see www.crncc.nihr.ac.uk

\section{PETER SELBY}

Director

JOHN SITZIA

Acting chief operating officer

\section{Reference}

1 Goodacre J. Nature Med 2010;16:158.

\section{National Institute for Health Research Clinical Research Network}

Editor - On entering medical school, we were taught that the most fundamental skill for a medical student to learn was how to elicit an accurate history from a patient, requiring communication skills, an inquisitive mind and above all, patience. Once the scene was set by the history, the examination would proceed to add depth, colour and clarity to the patient's story-board narrative. History taking should generate a hypothesis, which is either confirmed or refuted by the examination. This generates a working diagnosis, which leads to a minimum of appropriate tests to refine. After all, we can only run after we have learnt to walk.

Historically, this was what made medicine in Great Britain, great. Methodological reasoning and clinical acumen. Yet increasingly, we seem to have forgotten the works of William Occam, Reverend Bayes and Thomas Hutchinson, and have increasingly developed a 'test first, question later' mentality. We ignore the history, rush the examination and order a smörgåsbord of biochemical, haematological and radiological tests, fishing for a result and then treating it. We concentrate on the most serious or nebulous diagnosis, ignore the more common or more likely and often fail to consider a differential.

At the dark heart of this disintegration of medical practice in the UK is the fear of litigation and the perception of failure by 'missing something'. We prefer to believe in a seemingly flawless and objective 'test' rather than an imperfect and subjective 'judgement'. Making a rod for our own backs, we 'protect' the most inexperienced doctors from making judgements, expecting only those with more experience to do so, and in so doing de-skilling all concerned. Furthermore, a test might be positive but it is only of use and significance if we have a context in which to place it. Raised D-Dimer anyone?

Tests all have costs. For the organisation there are both financial and opportunity costs; for the patient there are costs in terms of morbidity (radiation exposure; complications of invasive investigation; hospital-acquired infection during the prolonged stay) and emotional harm, especially when putative diagnoses are raised which may result from false positive or negative tests. Inappropriate tests can also lead both to treatment that is unnecessary, and to more tests, often over a prolonged period (serial computed tomography scans following discovery of an incidental benign nodule) further increasing patient anxiety and potential morbidity.

Perhaps the biggest cost of ignoring the basic principles of the above approach to the patient is damage to our professionalism and to our profession per se. Doctors are trained to have the clinical skills to elicit the appropriate information. Judgement is then employed to weigh up that information effectively and efficiently to reach a diagnostic conclusion and make decisions about treatment.

The explosion of guidelines might be partly to blame. While they have their strengths, clinical guidelines do not foster a culture of the critical appraisal and evaluation of the usual disparate elements in the history and examination, which so often cloud diagnostic decision making. They are often rigid and straight-jacket thinking along specific lines; they are based on typical presentations, when in reality clinical medicine is rarely so straight forward. More importantly they are increasingly enforced by para-medical staff as rules not guidance. For a junior doctor to stray from a guideline leads to criticism and opprobrium from non-medical colleagues. To use guidelines, the critical appraisal skills for which a doctor has been trained are unnecessary. You don't have to be a doctor to use them.

Fundamentally, we all believe we hear the patient, but do we actually listen to what is said? We can diagnose a pulmonary embolus without touching the patient's bruised chest, cellulitis without asking if the patient's leg is normally swollen and red, and an acute coronary syndrome based only on a raised serum troponin concentration.

Perhaps we should bear in mind these commonsense maxims:

- Common things are common, but never say never.

- Treat acute things acutely and chronic things chronically.

- If a patient looks and feels well, they are well, despite what the tests say.

- If you allow them the time and space, the patient will tell you the diagnosis.

- If you hear the clap of hooves, it is probably not a unicorn.

We must embrace commonsense in medicine if we are to save our patients, our personal reputation, our profession and our NHS. How about a National Day for Commonsense in Medicine where the only available resource is our clinical skills?

We are all guilty of over-investigating to cover ourselves, just in case, to 'rule out' unlikely alternatives when the answer is staring us in the face and trying to tell us. All the examinations, research and courses in the world cannot teach commonsense; this must be seen first hand in practice, by our students and juniors and we must take the time to teach this and teach it well, for we reap what we sow.

DAVID WARRINER

Senior house office cardiology, Northern General Hospital, Sheffield

RICHARD TURNER

Specialist registrar respiratory medicine, Northern General Hospital, Sheffield

WILL SHIPPAM

House officer gastroenterology, Barnsley Hospital, Barnsley

\section{Teaching and learning on busy post-take ward rounds}

Editor - I read with interest the article by Graeme Dewhurst (Clin Med June 2010 pp 231-4). In the article, Dewhurst investigates those factors which are considered by junior 